\title{
Protein Kinase A Signaling Inhibits Iridophore Differentiation in Zebrafish
}

\author{
Cynthia D. Cooper ${ }^{1,2, *} \mathbb{D}$, Steve D. Erickson ${ }^{2}\left(\mathbb{D}\right.$, Scott Yin ${ }^{2}$, Trevor Moravec ${ }^{2}$, Brian Peh $^{2}$ and \\ Kevin Curran ${ }^{3}$ \\ 1 School of Molecular Biosciences, Washington State University Vancouver, Vancouver, WA 98686, USA \\ 2 College of Arts and Sciences, Washington State University Vancouver, Vancouver, WA 98686, USA; \\ stevederickson@gmail.com (S.D.E.); scottyin@yahoo.com (S.Y.); moravect@wsu.edu (T.M.); \\ brianpeh@comcast.net (B.P.) \\ 3 Department of Biology, University of San Diego, San Diego, CA 92110, USA; kevincurranuw@gmail.com \\ * Correspondence: cdcooper@wsu.edu; Tel.: +1-360-546-9342
}

Received: 10 August 2018; Accepted: 14 September 2018; Published: 26 September 2018

check for updates

\begin{abstract}
In zebrafish (Danio rerio), iridophores are specified from neural crest cells and represent a tractable system for examining mechanisms of cell fate and differentiation. Using this system, we have investigated the role of cAMP protein kinase A (PKA) signaling in pigment cell differentiation. Activation of PKA with the adenylyl cyclase activator forskolin reduces the number of differentiated iridophores in wildtype larvae, with insignificant changes to melanophore number. Inhibition of PKA with H89 significantly increases iridophore number, supporting a specific role for PKA during iridophore development. To determine the effects of altering PKA activity on iridophore and melanophore gene expression, we examined expression of iridophore marker $p n p 4 a$, melanophore marker mitfa, and the mitfa repressor foxd3. Consistent with our cell counts, forskolin significantly decreased pnp $4 a$ expression as detected by in situ hybridization and quantification of pnp $4 a+$ cells. Forskolin had the opposite effect on mitfa and foxd3 gene activity, increasing the area of expression. As mitfa/nacre mutants have extra iridophores as compared to wildtype larvae, we examined the function of mitfa during PKA-sensitive iridophore development. Forskolin treatment of mitfa/nacre mutants did significantly reduce the number of iridophores but to a lesser extent than that observed in treated wildtype larvae. Taken together, our data suggests that PKA inhibits iridophore development in a subset of iridophore precursors, potentially via a foxd3-independent pathway.
\end{abstract}

Keywords: iridophores; PKA signaling; forskolin; melanophores; differentiation; mitfa; pnp $4 a$

\section{Introduction}

Chromatophores, or pigment/light-reflecting cells, develop from neural crest cells, a population of cells that arise near the dorsal aspect of the developing vertebrate neural tube. Five types of chromatophores have been characterized in different organisms, including several species of fish. These cell types include black melanophores, yellow xanthophores, silver iridescent iridophores, white reflective leucophores, and red erythrophores (reviewed in [1]). Chromatophores promote temporary or permanent color change and are thus critical for an aquatic organism's ability to evade predators, to blend into their environments, or to find mates. Zebrafish have a subset of these chromatophores, including melanophores, xanthophores, and iridophores. Each cell type represents an excellent system for understanding cell fate decisions and subsequent differentiation. Melanophores are the best characterized and loss of function mutations in zebrafish microthalmia-associated transcription factor (mitfa), kit, oculocutaneous albinism 2 (oca2), and vacuolar protein sorting 11 (vps) genes promote phenotypes similar to those found in mammalian knockouts (i.e., loss of pigment cell precursors or 
under/hypopigmented melanocytes; [2-9]). Some pathways affecting melanocyte development also impact iridophore development, offering a separate system for understanding the activity or function of these pathways during cell differentiation. For example, the number of iridophores is impacted by several loss of function mutations in genes previously characterized in melanocytes, including oca 2 (increased iridophores), vps11 (decreased iridophores), and mitfa (increased iridophores) as compared to age-matched wildtype larvae. Additionally, Forkhead transcription factor foxd3 regulates the expression of mitfa and nucleoside phosphorylase gene, $p n p 4 a$, in a bipotent melanophore/iridophore precursor cell, suggesting that a population of cells exists with the capability of becoming either pigment cell type [10]. It remains unclear as to which intracellular signaling proteins control iridophore differentiation decisions. Here, we propose a role for protein kinase A (PKA) in inhibiting the iridophore differentiation program.

PKA signaling is tightly controlled by regulation of cAMP gradients. Adenylyl cyclases generate cAMP and phosphodiesterases-the latter degrades cAMP (reviewed in [11]). Upon activation by cAMP (via regulatory unit binding), protein kinase A catalytic units phosphorylate target proteins involved in several cellular processes, including cell differentiation and growth [12-15]. PKA signaling also functions in organelle trafficking within chromatophores. Melanophore melanin-producing organelles, melanosomes, are trafficked towards the cell periphery (dispersion) or nucleus (aggregation) in response to hormones such as cAMP-dependent melanocyte-stimulating and melanocyte-concentrating hormones, respectively [16]. Similarly, xanthophore pigment-producing organelles, xanthosomes, internalize their pigment granules in response to changes in light in a cAMPand phosphodiesterase-dependent manner [17]. Last, PKA signaling also regulates transcriptional programming important for neural crest cell fate choice in quail cell cultures, resulting in the induction of the melanocyte differentiation gene Mitf while simultaneously inducing expression of the RE-1 silencing transcription factor, a neuron-specific transcriptional repressor. These results were duplicated in zebrafish models where involvement of the Wnt signaling responsive transcription factor, CtBP2, was required for increased melanophore differentiation [18], suggesting a cross-talk between Wnt and PKA signaling. Thus, there is previous evidence of PKA serving as a switch to encourage differentiation of one lineage at the expense of a second. We explored a function for PKA signaling during iridophore development. Our data suggests that PKA signaling inhibits iridophore differentiation while promoting differentiation of melanophores in zebrafish larvae.

\section{Materials and Methods}

\subsection{Fish Rearing and Crosses}

Zebrafish work is approved by Washington State University Institutional Animal Care and Use Committee, Animal Subject Approval Form 03848. Wildtype fish were of the AB (ZDBGENO-960809-7) strain. Adult zebrafish were maintained on a $14 / 10 \mathrm{~h}$ light/dark cycle at $28.5^{\circ} \mathrm{C}$. Adult fish were maintained on a recirculating water system, fed dried/live diets, and checked daily. Embryos were acquired from natural crosses and grown at $28.5^{\circ} \mathrm{C}$ in embryo media until analysis. Embryos were staged according to characterized morphological criteria [19]. The following mutant alleles were used: nacre [7].

\subsection{Production of BACfoxd3::GFP Transgenic Zebrafish}

We modified a foxd3-containing bacterial artificial chromosome (BAC) clone by Escherichia coli-based homologous recombination [20]. BAC clone 137512 contains around $130 \mathrm{~kb}$ of sequence upstream and $40 \mathrm{~kb}$ downstream of foxd3 (http:/ / www.sanger.ac.uk/Projects/D_rerio/mapping.shtml). BAC clone 137512 was inserted into pTAR BAC 2.1. Following recombination, the modified BAC clone contained an egfp gene positioned at an endogenous start site. The accuracy of recombination was evaluated by PCR, sequencing, and by transient expression assays. BACfoxd3::EGFP faithfully recapitulated endogenous foxd3 expression in neural crest and other organs. To obtain a germline, we linearized BAC DNA with Not 1, injected linearized BAC DNA into zebrafish embryos, raised injected fish to adulthood, and screened their progeny for reporter gene expression. The germline transmission rate was $2 \%$. 


\subsection{Forskolin and $H-89$ Treatments}

Stocks of forskolin and H-89 were prepared in dimethyl sulfoxide (DMSO) at concentrations of 2.4 and $9.6 \mathrm{mM}$, respectively, and stored at $-20^{\circ} \mathrm{C}$ until use. Zebrafish were treated with $5 \mu \mathrm{M}$ forskolin (Sigma, St. Louis, MO, USA) diluted in embryo media beginning at 17-20 h post fertilization (hpf) unless otherwise indicated. At $48-50 \mathrm{hpf}$, forskolin-containing medium was removed, and fish were rinsed twice in fresh embryo media and incubated at $28.5^{\circ} \mathrm{C}$ until fixation. At the indicated timepoint, fish were fixed in $4 \%$ paraformaldehyde ( $4 \%$ PFA, diluted in phosphate-buffered saline, PBS), rinsed in PBS $+0.01 \%$ Tween (PBTw), and stored in PBTw (pre-in situ) until in situ hybridization/quantification or PBS $+0.01 \%$ Triton X-100 (PBTx) until antibody staining. Samples were stored in 50\% glycerol/PBS for analysis and to preserve fluorescence of antibody-stained samples. For H-89 (Sigma, St Louis, MO, USA) treatment, fish were treated with $25 \mu \mathrm{M}$ or $50 \mu \mathrm{M} \mathrm{H}-89$ in embryo media beginning at 17-20 hpf unless otherwise indicated. AT 48-50 hpf, H-89-containing media was removed, and fish were rinsed twice in fresh embryo media and incubated at $28.5{ }^{\circ} \mathrm{C}$ until fixation for quantification. $\mathrm{H}-89$ treated fish were fixed using $4 \%$ PFA and stored in PBTw until in situ hybridization analysis or 50\% glycerol/PBS until quantification.

\subsection{Cell Quantification and Statistics}

For iridophore quantification, a fiber optic light source was used to illuminate iridophores. Iridophores were counted using Nikon SMZ-1500 (Nikon Inc., Melville, NY, USA) or Leica M80 (Leica Microsystems, Inc., Buffalo Grove, IL, USA) stereomicroscopes. Unless otherwise indicated, cell counts include all (dorsal and ventral) trunk and tail iridophores. Fixed fish were used for quantification and $6 \%$ methyl cellulose was used to position fish for counting. Fish were rotated in methyl cellulose as necessary for viewing individual stripes/locations. For in situ hybridization analysis, fish were divided into "anterior body", "posterior body", and "tail" regions (see Figure D' for additional information). Anterior regions include tissue dorsal to rostral and caudal yolk boundaries, while posterior regions include tissue posterior to the caudal yolk boundary, ending at the end of the yolk extension. The tail region included tissue posterior to the yolk extension. Statistical tests performed for each experiment were the Student's $t$-test (Microsoft Excel 2007) or ANOVA (one- or two-way ANOVA; GraphPad Prism, San Diego, CA, USA). Data reported include a minimum of 8-10 samples per time point, with at least three replicates per experiment.

\subsection{In Situ Hybridization}

Embryos were fixed in $4 \%$ paraformaldehyde in phosphate-buffered saline at $\mathrm{pH} 7.2$, and processed using standard protocols [21]. Digoxigenin-labeled probes for mitfa [7], foxd3 [22,23], and pnp4a [10] have been previously described. All imaging was done using a Nikon SMZ-1500 stereomicroscope equipped with a Digital Sight DS-Ri1 Digital Camera. All images were processed for contrast, brightness, and color using Adobe Photoshop CS3 Extended Version 10.0 (Adobe Corporate Headquarters, San Jose, CA, USA).

\subsection{Antibody Staining and Confocal Imaging}

The following antibodies were used for immunohistochemistry at the indicated dilutions: mouse monoclonal anti-Green Fluorescent Protein (Invitrogen/ThermoFisher Scientific, Waltham, MA, USA), 1:500; rabbit anti-beta catenin (Pharmingen, San Diego, CA, USA), 1:100; anti-mouse (Alexa 488) and anti-rabbit (Alexa 568) secondary antibodies (Molecular Probes, Eugene, OR, USA) 1:500 or 1:750. Brightfield or incident lighting (epi-illumination from a fiber optic/ectopic light source) images were obtained on a Nikon dissecting microscope with a Spot RT Slider digital camera (Diagnostic Instruments, Grand Island, NY, USA) or a Nikon SMZ-1500 stereomicroscope with a Digital Sight DS-Ri1 Digital camera. Fluorescent confocal images were obtained on LSM 5 Pascal (Zeiss, Thornwood, NY, USA) or Olympus FV1000 laser scanning confocal microscopes. Images were equivalently processed for color balancing and brightness/contrast using Photoshop CS3 or CS4 (Adobe, San Jose, CA, USA) and formatted with Illustrator CS4 (Adobe, San Jose, CA, USA). 


\section{Results and Discussion}

\subsection{Effects of Protein Kinase A Activity Alter the Number of Differentiated Iridophores}

To examine the role of protein kinase A during iridophore development, we treated zebrafish embryos with forskolin, an adenylyl cyclase activator. Treatments commenced at 17-20 h post fertilization (hpf) when iridophores are specified from neural crest cells [10]. Following washout of forskolin at 2 days post fertilization (dpf), larvae were grown until $4 \mathrm{dpf}$ when they were fixed for iridophore quantification. At this timepoint, iridophores are positioned one cell per somite, making them easy to quantify. Using an incident light source, we were able to illuminate and observe individual iridophores in the dorsal and ventral stripes, beginning at the anterior trunk level. Imaging analysis indicates fewer iridophores in forskolin-treated individuals, especially in the ventral stripes (Figure 1A,B; white arrowheads indicate the ventral stripe). Quantification of iridophores in dorsal and ventral stripes confirms a significant reduction in both stripes, and a $38.4 \%$ reduction in total iridophores (Figure 1C, $p<0.05$ via Student's $t$-test). To ensure this reduction was not due to developmental delay of the larvae, we also quantified melanophores which develop at a similar time to iridophores in zebrafish (Figure 1D). Statistical analysis suggests that while melanophore number is slightly higher with forskolin treatment (as compared to controls), this increase is not significant (Student's $t$-test, $p>0.05$; we also noted no significant differences in dorsal or ventral stripe numbers). Normal somite shape and yolk extension patterning/development further suggest minimal impact on other tissues in forskolin-treated larvae.
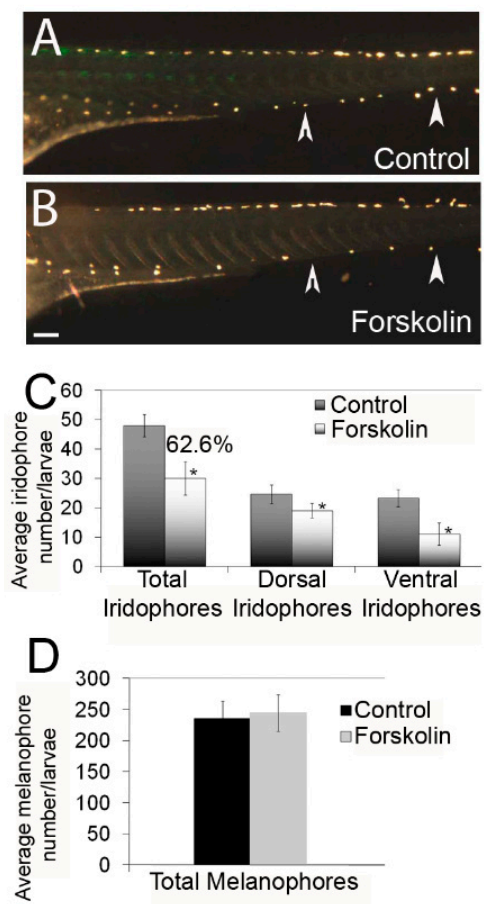

Figure 1. Forskolin treatment reduces iridophore number in zebrafish larvae at 4 days post fertilization (dpf). (A,B) Lateral images (using an incident lighting source) of larvae at $4 \mathrm{dpf}$ treated with $0.1 \%$ dimethyl sulfoxide (DMSO) or $5 \mu \mathrm{M}$ forskolin in embryo media. Forskolin-treated individuals have fewer iridophores, especially in the ventral stripe (arrowheads). Scale bar $=200 \mathrm{um}$ and applies to panels A and BC) Quantification of total, dorsal stripe, and ventral stripe iridophores ( $n=8-10$ larvae per condition). Forskolin treatment leads to a $38.4 \%$ reduction in differentiated iridophores $(62.6 \%$ of control number). (D) Quantification of melanophores following 0.1\% DMSO or $5 \mu \mathrm{M}$ forskolin treatment. Total melanophores include dorsal, lateral, and ventral stripes ( $n=8-10$ larvae per condition), showing no significant change following forskolin treatment. 
To confirm that forskolin effects were specific to protein kinase A, we analyzed the effects of the protein kinase A inhibitor, H-89 dihydrochloride (H-89), on iridophore number. We tested several concentrations to best understand the effects of distinct doses on iridophore development (Figure 2). Inhibition of protein kinase A via $25 \mu \mathrm{M}$ and $50 \mu \mathrm{M} \mathrm{H}-89$ treatment significantly increases iridophore number (one-way ANOVA with Bonferroni multiple comparison (* $p<0.05$ or ${ }^{* *} p<0.01$ )), consistent with our forskolin data. We do see a lower than expected number of iridophores at $100 \mu \mathrm{M}$, most likely due to developmental delay or toxicity with this dose. Taken together, this data suggests that protein kinase A functions to regulate the number of differentiated iridophores in developing zebrafish.

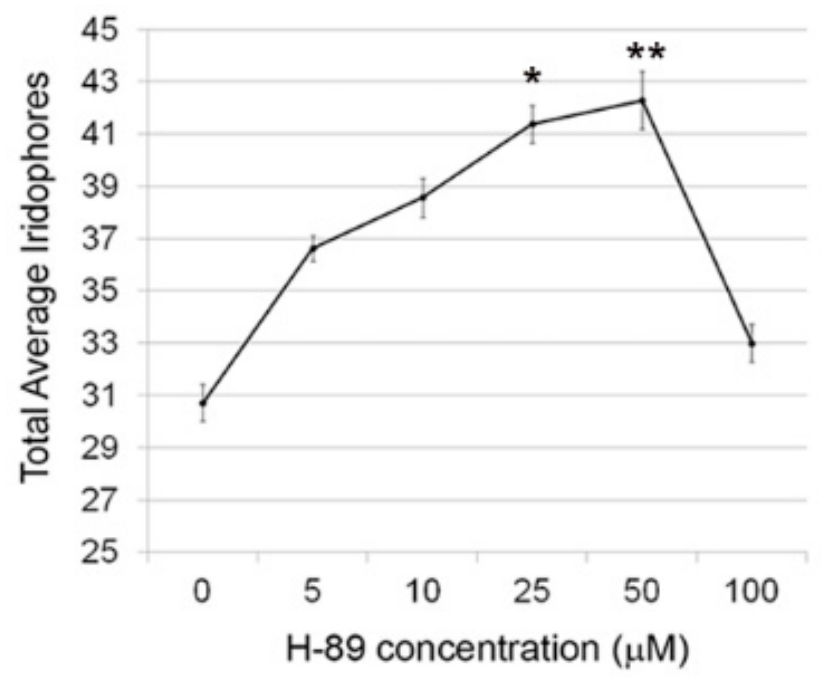

Figure 2. H89 treatment increases iridophore number in zebrafish larvae at $4 \mathrm{dpf}$. Quantification of iridophores following treatment of wildtype embryos with varying doses of the protein kinase A (PKA) inhibitor H89. One-way ANOVA with Bonferonni multiple comparison post-test indicates that doses of $25 \mu \mathrm{M}$ and $50 \mu \mathrm{M}$ are statistically significant $\left({ }^{*} p<0.05\right.$ or $\left.{ }^{* *} p<0.01\right)$.

\subsection{PKA Modulation Inhibits Iridophore Gene Expression}

Zebrafish iridophores are specified and then differentiate beginning at $20 \mathrm{~h}$ post fertilization (hpf), as detected by purine nucleoside phosphorylase (pnp4a) expression [10]. As PKA signaling functions during neuron differentiation [22], we hypothesized that PKA signaling regulates differentiation of the iridophore precursors, iridoblasts. To test this hypothesis, we examined pnp $4 a$ expression in forskolin and H89-treated larvae. Embryos were treated with forskolin, H89, or control (0.1\% DMSO) embryo medium beginning at $22 \mathrm{hpf}$. Larvae were fixed at $2 \mathrm{dpf}$ and analyzed via in situ hybridization for pnp $4 a$ expression. Forskolin treatment reduced the amount of $p n p 4 a+$ signal at $2 \mathrm{dpf}$ (posterior body; Figure 3A,B). Conversely, H89 individuals showed an increase in pnp4a signal (compare Figure 3C to Figure 3A). To determine if the number of $p n p 4 a+$ cells had significantly changed, we quantified the number of pnp $4 a+$ cells in the anterior body, posterior body, and tail. The number of $p n p 4 a+$ cells in posterior body/trunk regions of forskolin-treated larvae is significantly lower as compared to DMSO-treated controls (Figure 3D; ${ }^{* * *} p<0.0001$ via two way ANOVA with Bonferroni multiple comparisons analysis) and significantly higher with $\mathrm{H} 89$ treatment (** $p<0.01$ via two way ANOVA with Bonferroni multiple comparisons analysis) at $2 \mathrm{dpf}$. This data suggests that PKA activity inhibits iridophore differentiation by reducing pnp $4 a$ expression. 


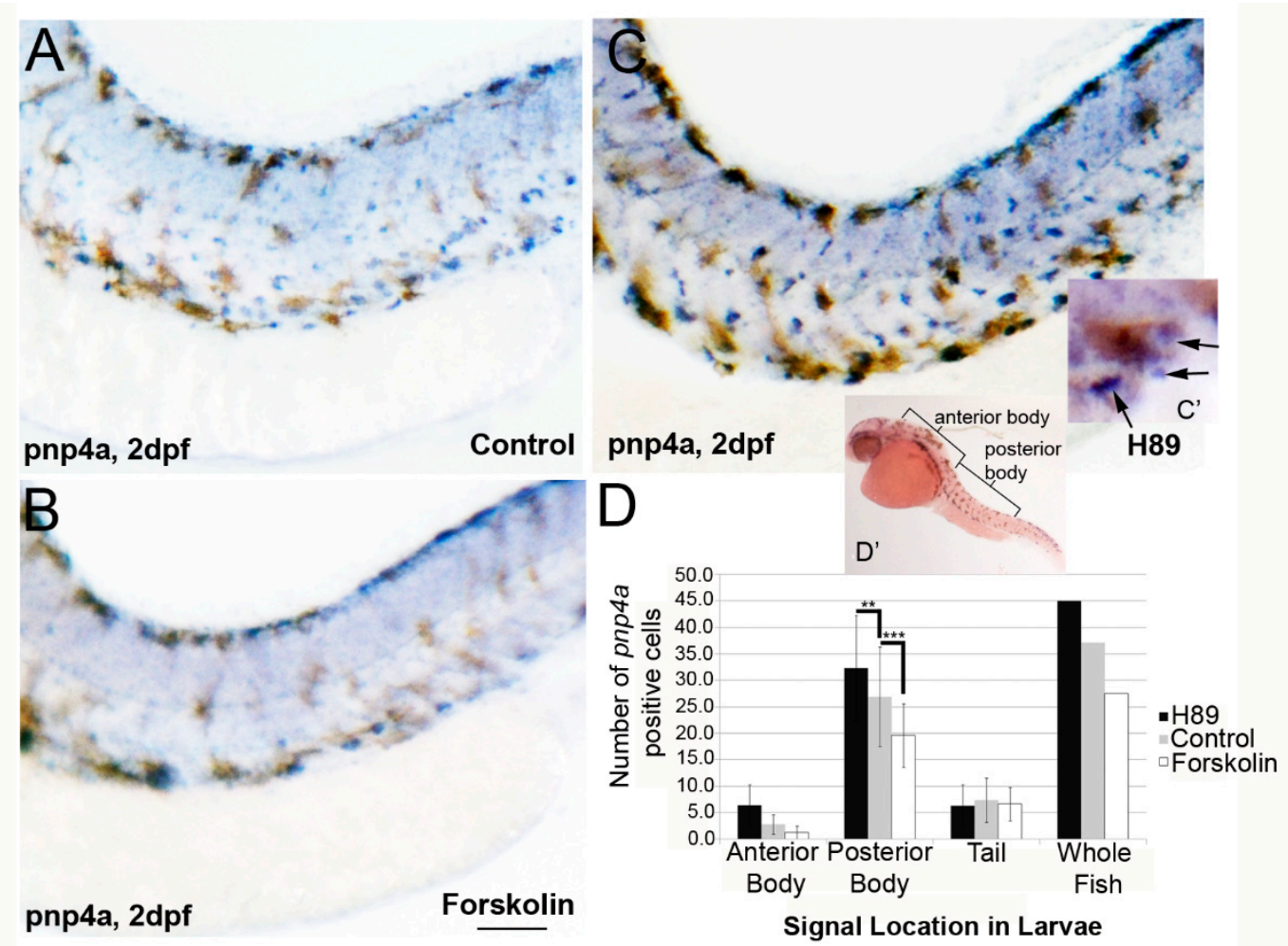

Figure 3. Forskolin treatment reduces iridoblast marker pnp4a expression. (A-C) Lateral brightfield images of larvae at $2 \mathrm{dpf}$ processed for in situ hybridization using pnp $4 a$ probes, following treatment with $0.1 \%$ DMSO (A), $5 \mu \mathrm{M}$ forskolin (B), or $25 \mu \mathrm{M} \mathrm{H}-89$ (C) in embryo media. Activation of adenylyl cyclase (and PKA signaling) reduces the levels of pnp $4 a$ expression, whereas inhibition of PKA has the converse effect (increased pnp $4 a$ expression). Scale bar $=200 \mathrm{um}$ andapplies to all images. $\left(\mathbf{C}^{\prime}\right)$ Representative image with black arrows showing blue/purple $p n p 4+$ cells included in panel D quantification. Brown melanophores were not included in counts. (D) Quantification of pnp4a+ cells confirms a significant reduction and increase in the number of $p n p 4 a+$ cells following forskolin and H-89 treatment, respectively ${ }^{* *} p<0.01$ and ${ }^{* *} p<0.0001$ via two-way ANOVA and Bonferonni multiple comparison analysis). ( $\left.\mathbf{D}^{\prime}\right)$ The quantified regions are labeled.

\section{3. mitfa Is Partially Required for the Forskolin-Dependent Reduction in Iridophore Number}

mitfa is required and sufficient for melanophore specification as indicated by nacre/mitfa zebrafish mutants, which do not develop melanophores [7]. Additionally, iridophores are present in excess in mitfa mutants as compared to wildtype control zebrafish $[7,10]$, suggesting that mitfa is critical for establishing correct iridophore number. Examination of simultaneous pnp $4 a$ and mitfa expression indicates co-expression of these gene products at $24 \mathrm{hpf}$ (but loss of co-expression by 50 hpf [10]). To examine the requirement of mitfa during PKA-sensitive iridophore differentiation, we first observed mitfa expression with or without PKA activation (forskolin treatment). Forskolin-treated larvae showed larger regions of mitfa expression posterior to the eye and in the dorsal tail as compared to DMSO treated controls (Figure 4A,B).

We next examined the effects of forskolin treatment on pnp $4 a$ expression in the dorsal trunk region of nacre/mitfa mutants. Again, treatment of wildtype embryos with forskolin caused a reduction in pnp $4 a$ expression at $2 \mathrm{dpf}$ as compared to controls (Figure 5A,B; consistent with previous results in Figure 3). Conversely, pnp4a expression was similar in DMSO/control and forskolin-treated nacre larvae at $2 \mathrm{dpf}$ (Figure 5C,D). To confirm that iridophore number was less impacted by PKA activity in nacre mutants with forskolin treatment, we quantified the number of iridophores in untreated and forskolin treated mitfa/nacre larvae at $4 \mathrm{dpf}$ (following a washout at $2 \mathrm{dpf}$ ). While wildtype/AB larvae at $4 \mathrm{dpf}$ 
showed a 38.4\% (See Figure 1) reduction in iridophores as compared to DMSO treated controls, nacre larvae showed a $22.2 \%$ reduction in iridophores (Figure 5E). Furthermore, only the forskolin-treated nacre mutant iridophore number was close to control numbers by $7 \mathrm{dpf}$ (control, $46.6 \pm 4.4$; forskolin $43.2 \pm 4.8$ ). Taken together, this data suggests that zebrafish larvae have a population of iridophores that are not sensitive to PKA in the absence of mitfa expression.
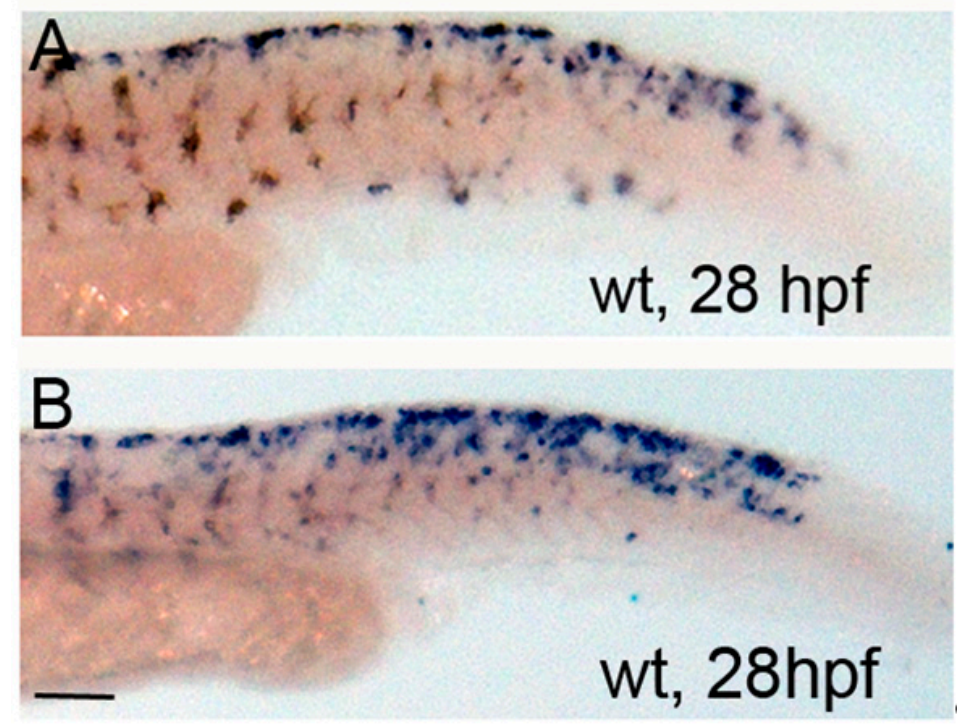

Figure 4. Forskolin treatment expands mitfa expression at 28 hpf. (A,B) Brightfield images of lateral tail regions of 28-h-old wildtype control and forskolin-treated embryos processed for in situ hybridization using mitfa probe. Note increased mitfa expression in the dorsal tail. Scale bar $=100 \mathrm{um}$ and applies to both images.

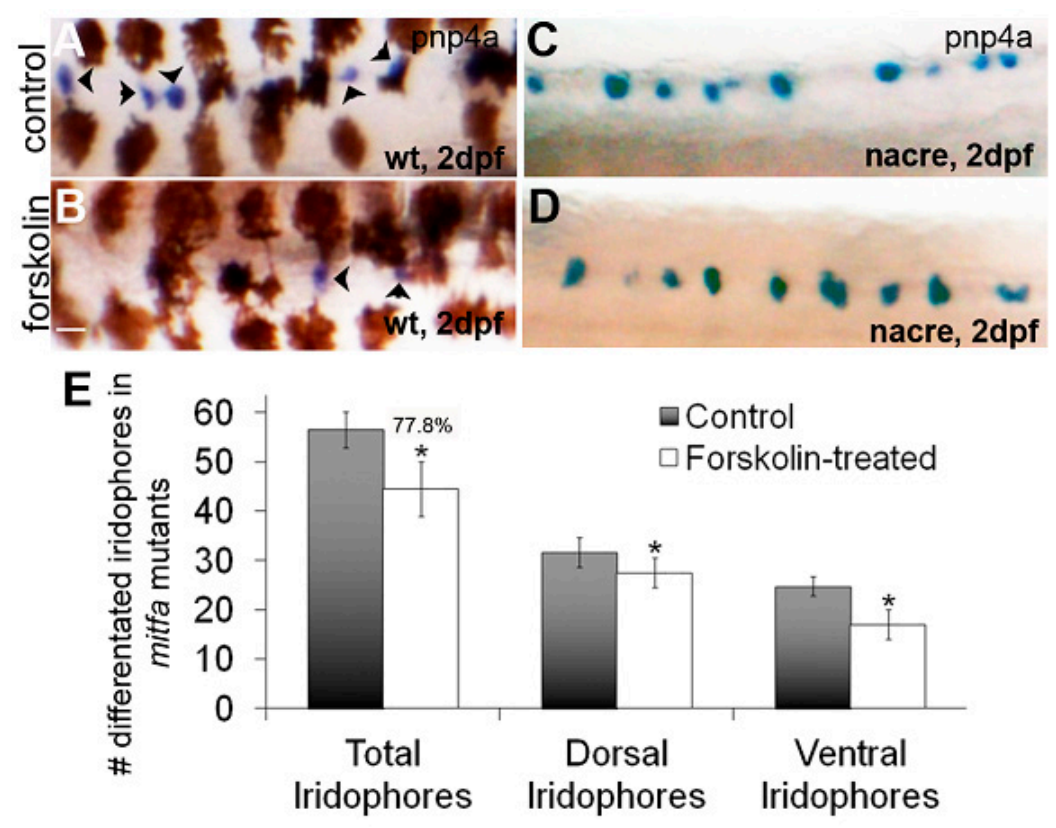

Figure 5. A subset of mitfa negative iridoblasts is resistant to PKA signaling. (A-D) Brightfield images of dorsal trunks of wildtype or nacre/mitfa mutant larvae at $2 \mathrm{dpf}$ treated with $0.1 \%$ DMSO or $5 \mu \mathrm{M}$ forskolin and processed for in situ hybridization using a pnp $4 a$ probe. A reduction in $p n p 4 a$ is detected in wildtype (as previously observed) but not in nacre/mitfa larvae. Scale bar $=100 \mathrm{um}$ and applies to all images. (E) Quantification of differentiated iridophores in $0.1 \%$ DMSO or $5 \mu \mathrm{M}$ forskolin-treated nacre/mitfa larvae. We note a significant, yet attenuated reduction in iridophores with mitfa loss of function $(22.2 \%$ reduction in iridophores or $77.8 \%$ of control). The reductions are significant via Student's $t$-test $(p<0.05)$. 


\subsection{Activation of PKA Expands Neural Crest Marker foxd3 Expression}

Forkhead box transcriptional repressor foxd 3 activity is important for specification of specific neural crest derivatives, including iridophores [23,24] and melanophores [25]. foxd3 is a well characterized marker for neural crest cells and presumptive iridophores. Consistently, foxd 3 is co-expressed with the iridophore marker pnp4a [10]. Additionally, evidence suggests that foxd3 represses its own transcription $[23,24]$. foxd3 intersects with several signaling pathways-including the ERBB3 signaling pathway in melanoma cells-via upregulation of specific signaling genes involved in cell proliferation and apoptosis [26,27] and directly represses mitfa expression-likely a necessary step in cells destined to become iridophores. Additionally, foxd3 is required for $p n p 4 a$ expression by developing iridophores [10]. We hypothesized that PKA inhibits foxd3, leading to increased mitfa expression/melanophore differentiation and decreased pnp $4 a$ expression/iridophore differentiation. To test this hypothesis, we used transgenic zebrafish expressing GFP under the control of the foxd3 promoter (about $130 \mathrm{~kb}$ from BAC clone 137512) and conducted in situ hybridization using a probe against foxd3. Analysis of GFP transgenics at $3 \mathrm{dpf}$ (Figure 6A-E) shows GFP expression in the expected foxd3-dependent locations, including the pineal gland (Figure $6 \mathrm{~B}$, arrowhead) and the skin (Figure $6 \mathrm{~B}, \mathrm{C}$ ), with presumptive iridophores indicated by arrows $[23,24,28]$. Higher magnification $(20 \times)$ confirms that GFP colocalizes with iridophores (Figure 6D-E). To determine the effects of forskolin treatment on foxd3 expression, we treated embryos with forskolin or DMSO control embryo media from 19 to $25 \mathrm{hpf}$, fixed them in 4\% PFA, and probed them using anti-GFP and anti-beta catenin antibodies. The analysis of beta catenin was added to better visualize cell boundaries. Unexpectedly, forskolin expanded the area(s) of foxd3 expression as detected by the GFP reporter (Figure 6F-G; arrowheads indicate neural crest positive pharyngeal arches) and in situ hybridization (Figure 6H-I). Dorsal head and trunk skin also showed a wider domain of foxd3 expression following forskolin treatment (arrows, Figure 6H-I). We also note similar locations of foxd3 expression, eye size, and pharyngeal arch development in forskolin-treated embryos as compared to control treated embryos, suggesting that $5 \mu \mathrm{M}$ forskolin has minimal impact on other developing tissues at this stage. Taken together, this data suggests that PKA does not inhibit iridophore differentiation by regulating foxd3 expression.

In summary, we have presented data supporting a role of PKA signaling in pigment cell differentiation. Specifically, we propose a model where PKA inhibits iridophore development while simultaneously promoting the development of a small subset of melanophores. PKA may also regulate melanin content as we noted changes in melanophore appearance and/or apparent melanin content with both treatments. Because the number of melanophores is so much greater than iridophores at the stages investigated, future studies will use a combination of qualitative and quantitative approaches to characterize the temporal and spatial requirements for PKA activity during iridophore and melanophore development. Although PKA does not inhibit foxd3 expression, it is possible that foxd3 protein activity is altered or that different mechanisms are at work in caudal versus trunk foxd3+ neural crest cells. Alternatively, PKA may activate foxd3 in cells not destined to become iridophores or melanophores (i.e., glia, neurons or other foxd3-dependent cells). Future studies will investigate the fate of these PKA-activated foxd3+ cells as well as the localization and phosphorylation status of Foxd3 protein. 

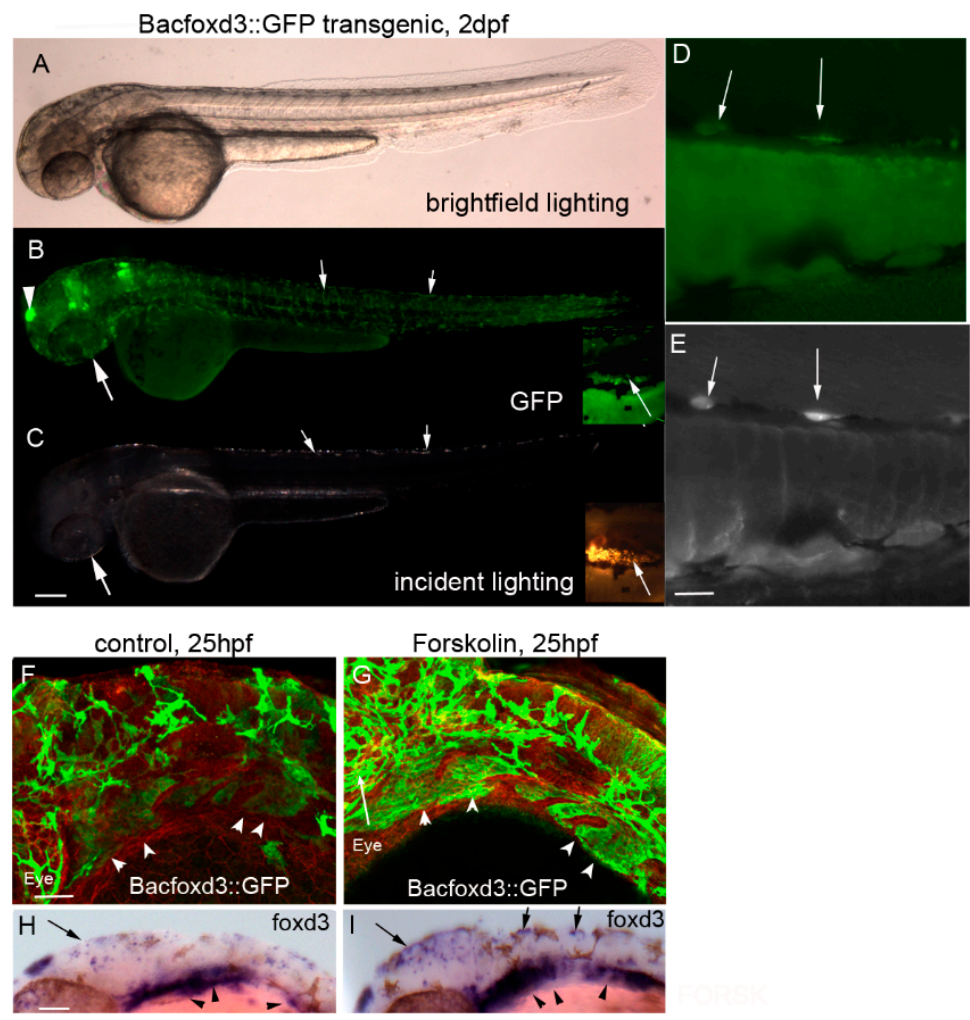

Figure 6. Forskolin increases expression of the mitfa transcriptional repressor foxd3. (A-C) $10 \times$ magnification of Bacfoxd3::GFP transgenic larvae at $2 \mathrm{dpf}$. Transgenic zebrafish express GFP in foxd3-dependent cells including the pineal gland (arrowhead) and presumptive iridophores (arrows). Scale bar in panel C $=200$ um and applies to panels A-C. (D-E) $20 \times$ magnification of BACfoxd3::GFP zebrafish. Arrows indicate cells co-expressing GFP and iridescence typically observed in iridophores. The scale bar in panel $\mathrm{E}=100 \mathrm{um}$ and applies to panels D and E. (F,G) $20 \times$ magnification of $25 \mathrm{hpf} 0.1 \%$ DMSO or $5 \mu \mathrm{M}$ forskolin-treated BACfoxd3::GFP transgenic fish processed for immunocytochemistry using anti-GFP and anti-beta catenin antibodies (red). Forskolin treatment leads to an increase in GFP (arrowheads indicate pharyngeal arches), indicating expanded domains of foxd3 expression. Scale bar in panel $\mathrm{F}=100 \mathrm{um}$ and applies to panels F and G. (H,I) $0.1 \%$ DMSO and $5 \mu \mathrm{M}$ forskolin-treated wildtype embryos processed for in situ hybridization using the foxd3 probe. Forskolin expands the areas of foxd3 expression, notably in the dorsal head and trunk regions (arrows) which are locations of presumptive iridophores, and in pharyngeal arch neural crest streams (arrowheads). Scale bar in panel $\mathrm{H}=100 \mathrm{um}$ and applies to panels $\mathrm{H}$ and $\mathrm{I}$.

\section{Conclusions}

Specification and differentiation of neural crest cells is a highly regulated process, relying on expression (or inhibition) of specific transcription factors and cell differentiation genes in response to environmental signals. Our work suggests that protein kinase A signaling turns on melanophore specific programming at the expense of iridophore programming in a subset of chromatophore precursor cells. This is the first study to implicate PKA signaling as an intracellular regulator of iridophore development.

Author Contributions: Conceptualization, C.D.C., S.D.E., and K.C.; Methodology, S.D.E., S.Y., T.M., B.P., K.C.; Formal Analysis, C.D.C., S.D.E., S.Y., T.M., B.P., K.C.; Investigation, S.D.E., S.Y., T.M., B.P., K.C.; Data Curation, C.D.C., S.D.E., S.Y., T.M., B.P., K.C.; Writing-Original Draft Preparation, C.D.C.; Writing-Review and Editing, C.D.C., S.D.E., S.Y., T.M., B.P., K.C.; Supervision, C.D.C.; Project Administration, C.D.C.; Funding Acquisition, C.D.C.; K.C. 
Funding: This research was supported (in part) by an American Cancer Society Institutional Research Grant (IRG-77-003-32 awarded to C.D.C), a research grant from the Melanoma Research Foundation (awarded to C.D.C.) and the Washington State University Vancouver faculty mini grant program (awarded to C.D.C.).

Acknowledgments: The authors are grateful to Alex Nechiporuk and David Raible for donating the BACfoxd3:: GFP clone.

Conflicts of Interest: The authors declare no conflict of interest.

\section{References}

1. Fujii, R. The regulation of motile activity in fish chromatophores. Pigment Cell Res. 2000, 13, 300-319. [CrossRef] [PubMed]

2. Beirl, A.J.; Linbo, T.H.; Cobb, M.J.; Cooper, C.D. oca2 regulation of chromatophore differentiation and number is cell type specific in zebrafish. Pigment Cell Melanoma Res. 2014, 27, 178-189. [CrossRef] [PubMed]

3. Clancey, L.F.; Beirl, A.J.; Linbo, T.H.; Cooper, C.D. Maintenance of Melanophore Morphology and Survival Is Cathepsin and vps11 Dependent in Zebrafish. PLoS ONE 2013, 8, e65096. [CrossRef] [PubMed]

4. Cooper, C.D.; Linbo, T.H.; Raible, D.W. Kit and foxd3 genetically interact to regulate melanophore survival in zebrafish. Dev. Dyn. 2009, 238, 875-886. [CrossRef] [PubMed]

5. Hultman, K.A.; Bahary, N.; Zon, L.I.; Johnson, S.L. Gene Duplication of the zebrafish kit ligand and partitioning of melanocyte development functions to kit ligand a. PLoS Genet. 2007, 3, e17. [CrossRef] [PubMed]

6. Lister, J.A.; Close, J.; Raible, D.W. Duplicate mitf genes in zebrafish: Complementary expression and conservation of melanogenic potential. Dev. Biol. 2001, 237, 333-344. [CrossRef] [PubMed]

7. Lister, J.A.; Robertson, C.P.; Lepage, T.; Johnson, S.L.; Raible, D.W. Nacre encodes a zebrafish microphthalmia-related protein that regulates neural-crest-derived pigment cell fate. Development 1999, 126, 3757-3767. [PubMed]

8. Parichy, D.M.; Rawls, J.F.; Pratt, S.J.; Whitfield, T.T.; Johnson, S.L. Zebrafish sparse corresponds to an orthologue of c-kit and is required for the morphogenesis of a subpopulation of melanocytes, but is not essential for hematopoiesis or primordial germ cell development. Development 1999, 126, 3425-3436. [PubMed]

9. Thomas, J.L.; Vihtelic, T.S.; denDekker, A.D.; Willer, G.; Luo, X.; Murphy, T.R.; Gregg, R.G.; Hyde, D.R.; Thummel, R. The loss of vacuolar protein sorting 11 (vps11) causes retinal pathogenesis in a vertebrate model of syndromic albinism. Investig. Ophthalmol. Vis. Sci. 2011, 52, 3119-3128. [CrossRef] [PubMed]

10. Curran, K.; Lister, J.A.; Kunkel, G.R.; Prendergast, A.; Parichy, D.M.; Raible, D.W. Interplay between Foxd3 and Mitf regulates cell fate plasticity in the zebrafish neural crest. Dev. Biol. 2010, 344, 107-118. [CrossRef] [PubMed]

11. Skroblin, P.; Grossmann, S.; Schafer, G.; Rosenthal, W.; Klussmann, E. Mechanisms of protein kinase A anchoring. Int. Rev. Cell Mol. Biol. 2010, 283, 235-330. [PubMed]

12. Ruehr, M.L.; Russell, M.A.; Bond, M. A-kinase anchoring protein targeting of protein kinase A in the heart. J. Mol. Cell Cardiol. 2004, 37, 653-665. [CrossRef] [PubMed]

13. Fimia, G.M.; Sassone-Corsi, P. Cyclic AMP signalling. J. Cell Sci. 2001, 114, 1971-1972. [PubMed]

14. Vandamme, J.; Castermans, D.; Thevelein, J.M. Molecular mechanisms of feedback inhibition of protein kinase A on intracellular cAMP accumulation. Cell Signal. 2012, 24, 1610-1618. [CrossRef] [PubMed]

15. Liang, W.; Ge, S.; Yang, L.; Yang, M.; Ye, Z.; Yan, M.; Du, J.; Luo, Z. Ginsenosides Rb1 and Rg1 promote proliferation and expression of neurotrophic factors in primary Schwann cell cultures. Brain Res. 2010, 1357, 19-25. [CrossRef] [PubMed]

16. Sheets, L.; Ransom, D.G.; Mellgren, E.M.; Johnson, S.L.; Schnapp, B.J. Zebrafish melanophilin facilitates melanosome dispersion by regulating dynein. Curr. Biol. 2007, 17, 1721-1734. [CrossRef] [PubMed]

17. Oshima, N.; Nakata, E.; Ohta, M.; Kamagata, S. Light-induced pigment aggregation in xanthophores of the medaka, Oryzias latipes. Pigment Cell Res. 1998, 11, 362-367. [CrossRef] [PubMed]

18. Liang, H.; Fekete, D.M.; Andrisani, O.M. CtBP2 downregulation during neural crest specification induces expression of Mitf and REST, resulting in melanocyte differentiation and sympathoadrenal lineage suppression. Mol. Cell Biol. 2011, 31, 955-970. [CrossRef] [PubMed] 
19. Kimmel, C.B.; Ballard, W.W.; Kimmel, S.R.; Ullmann, B.; Schilling, T.F. Stages of Embryonic Development of the Zebrafish. Dev. Dyn. 1995, 203, 253-310. [CrossRef] [PubMed]

20. Zhang, Y.; Buchholz, F.; Muyrers, J.P.; Stewart, A.F. A new logic for DNA engineering using recombination in Escherichia coli. Nat. Genet. 1998, 20, 123-128. [CrossRef] [PubMed]

21. Thisse, C.; Thisse, B.; Schilling, T.F.; Postlethwait, J.H. Structure of the zebrafish snail1 gene and its expression in wild-type, spadetail and no tail mutant embryos. Development 1993, 119, 1203-1215. [PubMed]

22. Wang, S.; Li, Z.; Shen, H.; Zhang, Z.; Yin, Y.; Wang, Q.; Zhao, X.; Ji, J. Quantitative Phosphoproteomic Study Reveals that Protein Kinase A Regulates Neural Stem Cell Differentiation Through Phosphorylation of Catenin Beta-1 and Glycogen synthase Kinase 3ß. Stem Cells 2016, 34, 2090-2101. [CrossRef] [PubMed]

23. Lister, J.A.; Cooper, C.; Nguyen, K.; Modrell, M.; Grant, K.; Raible, D.W. Zebrafish Foxd3 is required for development of a subset of neural crest derivatives. Dev. Biol. 2006, 290, 92-104. [CrossRef] [PubMed]

24. Stewart, R.A.; Arduini, B.L.; Berghmans, S.; George, R.E.; Kanki, J.P.; Henion, P.D.; Look, A.T. Zebrafish foxd3 is selectively required for neural crest specification, migration and survival. Dev. Biol. 2006, 292, $174-188$. [CrossRef] [PubMed]

25. Curran, K.; Raible, D.W.; Lister, J.A. Foxd3 controls melanophore specification in the zebrafish neural crest by regulation of Mitf. Dev. Biol. 2009, 332, 408-417. [CrossRef] [PubMed]

26. Abel, E.V.; Aplin, A.E. FOXD3 is a mutant B-RAF-regulated inhibitor of G(1)-S progression in melanoma cells. Cancer Res. 2010, 70, 2891-2900. [CrossRef] [PubMed]

27. Abel, E.V.; Basile, K.J.; Kugel, C.H., 3rd; Witkiewicz, A.K.; Le, K.; Amaravadi, R.K.; Karakousis, G.C.; Xu, X.; Xu, W.; Schuchter, L.M.; et al. Melanoma adapts to RAF/MEK inhibitors through FOXD3-mediated upregulation of ERBB3. J. Clin. Investig. 2013, 123, 2155-2168. [CrossRef] [PubMed]

28. Gilmour, D.T.; Maischein, H.M.; Nusslein-Volhard, C. Migration and function of a glial subtype in the vertebrate peripheral nervous system. Neuron 2002, 34, 577-588. [CrossRef]

(C) 2018 by the authors. Licensee MDPI, Basel, Switzerland. This article is an open access article distributed under the terms and conditions of the Creative Commons Attribution (CC BY) license (http:/ / creativecommons.org/licenses/by/4.0/). 